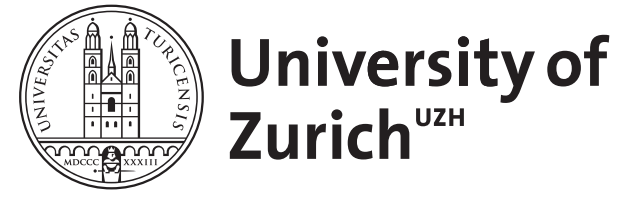

\title{
Dissipative continuous Euler flows
}

De Lellis, Camillo ; et al

\begin{abstract}
We show the existence of continuous periodic solutions of the 3D incompressible Euler equations which dissipate the total kinetic energy.
\end{abstract}

DOI: https://doi.org/10.1007/s00222-012-0429-9

Posted at the Zurich Open Repository and Archive, University of Zurich ZORA URL: https://doi.org/10.5167/uzh-85554

Journal Article

Published Version

Originally published at:

De Lellis, Camillo; et al (2013). Dissipative continuous Euler flows. Inventiones Mathematicae, 193(2):377407.

DOI: https://doi.org/10.1007/s00222-012-0429-9 


\section{Dissipative continuous Euler flows}

\section{Camillo De Lellis • László Székelyhidi Jr.}

Received: 9 February 2012 / Accepted: 14 October 2012 / Published online: 25 October 2012 (c) Springer-Verlag Berlin Heidelberg 2012

Abstract We show the existence of continuous periodic solutions of the 3D incompressible Euler equations which dissipate the total kinetic energy.

\section{Introduction}

In what follows $\mathbb{T}^{3}$ denotes the 3-dimensional torus, i.e. $\mathbb{T}^{3}=\mathbb{S}^{1} \times \mathbb{S}^{1} \times \mathbb{S}^{1}$. In this note we prove the following theorem.

Theorem 1.1 Assume e $:[0,1] \rightarrow \mathbb{R}$ is a positive smooth function. Then there is a continuous vector field $v: \mathbb{T}^{3} \times[0,1] \rightarrow \mathbb{R}^{3}$ and a continuous scalar field $p: \mathbb{T}^{3} \times[0,1] \rightarrow \mathbb{R}$ which solve the incompressible Euler equations

$$
\left\{\begin{array}{l}
\partial_{t} v+\operatorname{div}(v \otimes v)+\nabla p=0 \\
\operatorname{div} v=0
\end{array}\right.
$$

in the sense of distributions and such that

$$
e(t)=\int|v|^{2}(x, t) d x \quad \forall t \in[0,1] .
$$

C. De Lellis $(\bowtie)$

Institut für Mathematik, Universität Zürich, 8057 Zürich, Switzerland e-mail: camillo.delellis@math.uzh.ch

L. Székelyhidi Jr.

Institut für Mathematik, Universität Leipzig, 04103 Leipzig, Germany e-mail: laszlo.szekelyhidi@ math.uni-leipzig.de 
Obviously, if we choose a strictly decreasing function $e(t)$, Theorem 1.1 yields continuous solutions of the incompressible Euler equations which "dissipate" the total kinetic energy $\frac{1}{2} \int|v|^{2}(x, t) d x$. This is not possible for $C^{1}$ solutions: in that case one can multiply the first equation in (1) by $v$ to derive

$$
\partial_{t} \frac{|v|^{2}}{2}+\operatorname{div}\left(u\left(\frac{|u|^{2}}{2}+p\right)\right)=0 .
$$

Integrating this last identity in $x$ we then conclude

$$
\frac{d}{d t} \int_{\mathbb{T}^{3}} \frac{|v|^{2}}{2}(x, t) d x=0 .
$$

Theorem 1.1 shows therefore that this formal computation cannot be justified for distributional solutions, even if they are continuous. The pair $(v, p)$ in Theorem 1.1 solves (1) in the following sense:

$$
\int_{0}^{1} \int_{\mathbb{T}^{3}}\left(\partial_{t} \varphi \cdot v+\nabla \varphi: v \otimes v+p \operatorname{div} \varphi\right) d x d t=0
$$

for all $\varphi \in C_{c}^{\infty}\left(\mathbb{T}^{3} \times(0,1) ; \mathbb{R}^{3}\right)$ and

$$
\int_{0}^{1} \int_{\mathbb{T}^{3}} v \cdot \nabla \psi d x d t=0 \quad \text { for all } \psi \in C_{c}^{\infty}\left(\mathbb{T}^{3} \times(0,1)\right) .
$$

Remark 1 In the usual definition of weak solution, (4) is replaced by the same condition for divergence free test fields: therefore $p$ disappears from the identity. With this alternative definition, for every weak solution $v$ which belongs to $L^{2}$ a corresponding pressure field can then be recovered using

$$
-\Delta p=\operatorname{div} \operatorname{div}(v \otimes v) .
$$

$p$ is then determined up to an arbitrary function of $t$ : this arbitrariness can be overcome by imposing, for instance, $\int p(x, t) d x=0$. However, as it is wellknown, (5) and the continuity of $v$ does not guarantee the continuity of $p$.

\subsection{Onsager's conjecture}

The possibility that weak solutions might dissipate the total kinetic energy has been considered for a rather long time in the fluid dynamics literature: this phenomenon goes under the name of "anomalous dissipation". In fact, to our knowledge, the existence of dissipative solutions was considered for the first time by Lars Onsager in his famous 1949 note about statistical hydrodynamics, see [18]. In that paper Onsager conjectured that 
(a) $C^{0, \alpha}$ solutions are energy conservative when $\alpha>\frac{1}{3}$;

(b) There exist dissipative solutions with $C^{0, \alpha}$ regularity for any $\alpha<\frac{1}{3}$ (note that, though Onsager's definition of "weak solution" is, strictly speaking, different from the one given above, it can be easily shown that the two concepts are equivalent).

The first part of the conjecture, i.e. assertion (a), has been shown by Eyink in [11] and by Constantin, E and Titi in [3]. The proof of the last paper amounts to give a rigorous justification of the formal computation sketched above and leading to (3): this is done via a suitable regularization of the equation and some careful commutator estimates. The second part of the conjecture, i.e. statement (b), is still widely open. A first result in that direction was the groundbreaking work of Scheffer [20] which proved the existence of a compactly supported nontrivial weak solution in $\mathbb{R}^{2} \times \mathbb{R}$. A different construction of the existence of a compactly supported nontrivial weak solution in $\mathbb{T}^{2} \times \mathbb{R}$ was then given by Shnirelman in [21]. In both cases the solutions are only square summable (as a function of both space and time variables): it is therefore not clear whether there are intervals of time in which the total kinetic energy is a monotone function (indeed it is not even clear whether these solutions belong to the energy space $L_{t}^{\infty}\left(L_{x}^{2}\right)$ ). The first proof of the existence of a solution for which the total kinetic energy is a monotone decreasing function has been given by Shnirelman in [22]. Shnirelman's example is only in the energy space $L^{\infty}\left(\left[0, \infty\left[, L^{2}\left(\mathbb{R}^{3}\right)\right)\right.\right.$.

\section{$1.2 h$-principle}

Our previous work $[6,7]$ showed the existence of dissipative solutions for which both pressure and velocity are bounded. Besides the obvious improvement (and the discovery of quite severe counterexamples to the uniqueness of admissible solutions, both for incompressible and compressible Euler), in this work we introduced a new point of view in the subject, highlighting connections to other counterintuitive solutions of (mainly geometric) systems of partial differential equations: in geometry these solutions are, according to Gromov, instances of the $h$-principle. In particular Onsager's conjecture bears striking similarities with the rigidity and flexibility properties of isometric embeddings of Riemannian manifolds, pioneered by the celebrated work of Nash [17]. Indeed, results of the same flavor as statements (a) and (b) can be proved in the case of isometric embeddings (see for instance [4] and the references therein): in comparing Onsager's conjecture and these results, the reader should take into account that, in this analogy, the velocity field of the Euler equations corresponds to the differential of the embedding in the isometric embedding problem. All these aspects (and further developments for some PDEs in fluid dynamics inspired by our work) are surveyed in the note [8]. See also [1, 5, 23, 26-28]. 


\subsection{Comments on the proof}

As in all applications of convex integration, the solution of (1) is constructed by an iteration scheme: at each stage of this iteration we produce an "almost solution" which solves Euler with an additional error term. We name the resulting "perturbed" system of equations Euler-Reynolds system, since the error term has the typical form of the so-called Reynolds stress in the fluid dynamics literature (see [8] for an informal discussion of this point). This error term converges to 0 , while the sequence of almost solutions converge to an exact solution, uniformly in $C^{0}$. At each stage the new approximate solution is generated from the previous one by adding fast oscillatory perturbations. Being oscillatory, the linear terms in the perturbation are negligible whereas the nonlinear (quadratic) term is the leading order, which should cancel the previous error.

Although this general underlying philosophy is the same as in our previous papers $[6,7]$, in the actual proof we need to substantially depart from the previous strategy and introduce several new ideas.

(1) First of all, recall that in [6] we rewrite the Euler equations as a differential inclusion and thus we are able to apply some of the machinery developed in the context of Lipschitz differential inclusions. In particular, in all our results for bounded weak solutions there are two routes: a Baire category approach and a more direct convex integration approach. In fact these approaches are in some sense equivalent, see [25].

For continuous solutions the Baire category approach does not work as there is no way to extract a uniform continuity estimate for approximating sequences. In this paper we are therefore forced to abandon this, essentially "soft" functional analytic technique in favor of a more "hard" PDE approach based on Schauder estimates and oscillatory integrals. This seems to be entirely new in the context of convex integration, even compared to the original proof of Nash [17] for rough isometric embeddings.

(2) In all applications so far of convex integration to fluid mechanics [1, 5, $8,23,26-28]$ a key starting point is a plane-wave analysis, in order to identify compatible plane-waves that will be used in the subsequent construction. Indeed, the use of suitably localized plane-waves for an iteration scheme goes back to the work of Nash [17] and has been widely generalized by Gromov [13] as the technique of convex integration-the simple reason being that one-dimensional oscillations can be "integrated", hence the name convex integration. In the scheme of Nash simple plane-waves suffice to produce a $C^{1}$ solution. Nevertheless, it is crucial here that one has an extra dimension (the codimension of the range) to absorb linear errors.

For the Euler equations we have no extra dimension and simple planewaves do not work for continuous solutions. This leads to two points of departure from existing schemes: we use genuinely multi-dimensional building 
blocks, the Beltrami flows, and we retain a linear "transport term" in the iteration, which needs to be controlled with new ideas.

(3) As mentioned above, the main building blocks of our iteration scheme are Beltrami flows, which are truly three-dimensional oscillations. The issue of going beyond one-dimensional oscillations has been raised by Gromov (p. 219 of [13]) as well as Kirchheim-Müller-Šverák (p. 52 of [15]), but as far as we know, there have been no such examples in the literature so far. As a result of using Beltrami flows, the final result of our iteration scheme is the superposition of infinitely many (perturbed) and weakly interacting Beltrami flows. Curiously, the idea that turbulent flows can be understood as a superposition of Beltrami flows has been proposed almost 30 years ago in the fluid dynamics literature: see the work of Constantin and Majda [2]. Indeed, it was Peter Constantin who suggested to us to try Beltrami flows in a convex integration scheme.

(4) The "transport term" is perhaps the most important new aspect of our scheme in comparison with Nash. This arises, roughly speaking, as the linearization of the first equation in (1): this term is typical of an evolution equation, whereas, instead, the equations for isometric embeddings are "static". Moreover, the linear terms in the Nash scheme are set to zero by choosing suitable coordinates and working with the extra dimension. At a first glance this transport term makes it impossible to use a scheme like the one of Nash to prove Theorem 1.1. To overcome this obstruction we need to introduce a phase-function that acts as a kind of discrete Galilean transformation of the (stationary) Beltrami flows, and to introduce an "intermediate" scale along each iteration step on which this transformation acts.

(5) A further difference to the approach in $[6,7]$ is that we cannot use simple potentials to generate space-time localized versions of the Beltrami flows. One reason is that, in a sense, we cannot simply "integrate" Beltrami flows. More importantly, a space-time cutoff would result in error terms which cannot be controlled in $C^{0}$. In order to overcome this issue we introduce a "corrector term" to the main perturbation. This corrector term is not "explicit": it is determined by solving some appropriate elliptic equations.

(6) In comparing Theorem 1.1 to the main result in [7] an important difference is that, whereas in the bounded case $e$ is a function of $(x, t)$, here it is a function of $t$ only. In other words, whereas for bounded solutions we can prescribe $(x, t) \mapsto|v(x, t)|$, for continuous solutions we are only able to prescribe $t \mapsto \int|v(x, t)|^{2} d x$. As a consequence, our solutions in this paper cannot deal with the local energy inequality (cf. with the discussion in Sect. 3.1 of [8]). We do not know whether this is just a technical issue or a new feature of continuous solutions.

As a minor comment we remark that obviously the smoothness of $e$ in Theorem 1.1 can be relaxed, but we do not pursue this issue here. Moreover, the same theorem can be proved if we replace $[0,1]$ with $[0, \infty[:$ in this case 
we require in addition that $e$ and its derivatives are uniformly bounded and that there is a positive constant $c_{0}$ with $e \geq c_{0}$.

(7) In Theorem 1.1 our aim was to construct continuous weak solutions. In particular we did not address issues concerning the initial-value problem. Thus, setting aside the difference between bounded and continuous solutions, our Theorem 1.1 is weaker than the results proved in [7]. In particular, in this paper we do not prove any non-uniqueness statement.

However, this is a typical way to proceed with problems involving the $h$ principle. In general one may distinguish two aspects: a local and a global one. In geometric situations the local one is typically a differential constraint whereas the global one is topological (cf. [10]). The flexibility (in other words the lack of uniqueness) that one observes in instances of the $h$-principle is tied to the specifics of the local aspect. Thus, our Theorem 1.1 deals exclusively with the "local" aspect for the Euler equations, whereas a possible analogue of the global aspect would be the imposition of an initial data, possibly together with an admissibility condition (as in [7]). In subsequent papers we plan to address such "global" issues (e.g. initial data, compactly supported ancient solutions, etc.).

\section{Setup and plan of the paper}

The proof of Theorem 1.1 will be achieved through an iteration procedure. Along the iteration the maps will be "almost solutions" of the Euler equations. To measure "how far" a solenoidal field is from being a solution of incompressible Euler we introduce a system of differential equations which we call Euler-Reynolds system. The name is justified by the fact that the matrixfield $R$ is a well known object in the theory of turbulence, called "Reynolds stress" (cf. with [8] and the references therein). In what follows $\mathcal{S}_{0}^{3 \times 3}$ denotes the vector space of symmetric trace-free $3 \times 3$ matrices. Moreover we will, as it is usual, use the word smooth for $C^{\infty}$ maps.

Definition 2.1 Assume $v, p, \stackrel{\circ}{R}$ are smooth functions on $\mathbb{T}^{3} \times[0,1]$ taking values, respectively, in $\mathbb{R}^{3}, \mathbb{R}, \mathcal{S}_{0}^{3 \times 3}$. We say that they solve the EulerReynolds system if

$$
\left\{\begin{array}{l}
\partial_{t} v+\operatorname{div}(v \otimes v)+\nabla p=\operatorname{div} \stackrel{\circ}{R}, \\
\operatorname{div} v=0 .
\end{array}\right.
$$

We are now ready to state the main proposition of this paper, of which Theorem 1.1 is a simple corollary.

Proposition 2.2 Let e be as in Theorem 1.1. Then there are positive constants $\eta$ and $M$ with the following property. 
Let $\delta \leq 1$ be any positive number and $(v, p, \stackrel{\circ}{R})$ a smooth solution of the Euler-Reynolds system (6) such that

$$
\frac{3 \delta}{4} e(t) \leq e(t)-\int|v|^{2}(x, t) d x \leq \frac{5 \delta}{4} e(t) \quad \forall t \in[0,1]
$$

and

$$
\sup _{x, t}|\stackrel{\circ}{R}(x, t)| \leq \eta \delta .
$$

Then there is a second smooth triple $\left(v_{1}, p_{1}, \stackrel{\circ}{R}_{1}\right)$ which solves as well the Euler-Reynolds system and satisfies the following estimates:

$$
\begin{aligned}
& \frac{3 \delta}{8} e(t) \leq e(t)-\int\left|v_{1}\right|^{2}(x, t) d x \leq \frac{5 \delta}{8} e(t) \quad \forall t \in[0,1], \\
& \sup _{x, t}\left|\stackrel{R}{1}_{1}(x, t)\right| \leq \frac{1}{2} \eta \delta, \\
& \sup _{x, t}\left|v_{1}(x, t)-v(x, t)\right| \leq M \sqrt{\delta}
\end{aligned}
$$

and

$$
\sup _{x, t}\left|p_{1}(x, t)-p(x, t)\right| \leq M \delta .
$$

As already mentioned, Theorem 1.1 follows immediately from Proposition 2.2.

Proof of Theorem 1.1 We start by setting $v_{0}=0, p_{0}=0, \stackrel{\circ}{R}_{0}=0$ and $\delta:=1$. We then apply Proposition 2.2 iteratively to reach a sequence $\left(v_{n}, p_{n}, \stackrel{\circ}{R}_{n}\right)$ which solves (6) and such that

$$
\begin{aligned}
& \frac{3}{4} \frac{e(t)}{2^{n}} \leq e(t)-\int\left|v_{n}\right|^{2}(x, t) d x \leq \frac{5}{4} \frac{e(t)}{2^{n}} \quad \text { for all } t \in[0,1] \\
& \sup _{x, t}\left|\stackrel{R}{R}_{n}(x, t)\right| \leq \frac{\eta}{2^{n}} \\
& \sup _{x, t}\left|v_{n+1}(x, t)-v_{n}(x, t)\right| \leq M \sqrt{\frac{1}{2^{n}}} \\
& \sup _{x, t}\left|p_{n+1}(x, t)-p_{n}(x, t)\right| \leq \frac{M}{2^{n}} .
\end{aligned}
$$

Then $\left\{v_{n}\right\}$ and $\left\{p_{n}\right\}$ are both Cauchy sequences in $C\left(\mathbb{T}^{3} \times[0,1]\right)$ and converge uniformly to two continuous functions $v$ and $p$. Similarly $\stackrel{\circ}{R}_{n}$ converges 
uniformly to 0 . Moreover, by (13)

$$
\int_{\mathbb{T}^{3}}|v|^{2}(x, t) d x=e(t) \quad \forall t \in[0,1] .
$$

Passing into the limit in (6) we therefore conclude that $(v, p)$ solves (1).

\subsection{Construction of $v_{1}$}

The rest of the paper will be dedicated to prove Proposition 2.2. The construction of the map $v_{1}$ consists of adding two perturbations to $v$ :

$$
v_{1}=v+w_{o}+w_{c}=: v+w .
$$

To specify the form of the perturbation $w_{o}$, which is a highly oscillatory function and for which we give a rather explicit formula, we need several ingredients. The vectorfield $v+w_{o}$ is not in general divergence free. Therefore we add the correction $w_{c}$ to restore this condition. Having added the correction, the main focus will then be on finding maps $\stackrel{\circ}{R}_{1}$ and $p_{1}$ with the desired estimate and such that

$$
\partial_{t} v_{1}+\operatorname{div}_{x}\left(v_{1} \otimes v_{1}\right)+\nabla p_{1}=\operatorname{div}_{x} \stackrel{\circ}{R}_{1} .
$$

The perturbation $w_{o}$ will depend on two parameters, $\mu$ and $\lambda$, which will satisfy the following conditions

$$
\lambda, \mu, \frac{\lambda}{\mu} \in \mathbb{N} .
$$

In order to achieve the estimates, $\lambda$ and $\mu$ will be chosen quite large, depending on appropriate norms of $v$. As already mentioned, the building blocks for the perturbation $w_{o}$ are Beltrami flows. In order to give the formula leading to the definition of $w_{o}$ we must, therefore, study closer the particular "geometry" of these flows. This will be done in the next section. We will then be ready to define the perturbations $w_{o}$ and $w_{c}$ : this task will be accomplished in Sect. 4 where we also prescribe the constants $\eta$ and $M$ of the estimates in Proposition 2.2. After recalling some classical Schauder theory in Sect. 5, in the Sects. 6 and 7 we will prove the relevant estimates of the various terms involved in the construction, in terms of the parameters $\lambda$ and $\mu$. The choice of these parameters will be finally specified in Sect. 8, where we conclude the proof of Proposition 2.2.

\section{Geometric preliminaries}

In this paper we denote by $\mathbb{R}^{n \times n}$, as usual, the space of $n \times n$ matrices, whereas $\mathcal{S}^{n \times n}$ and $\mathcal{S}_{0}^{n \times n}$ denote, respectively, the corresponding subspaces 
of symmetric matrices and of trace-free symmetric matrices. The $3 \times 3$ identity matrix will be denoted with Id. For definitiveness we will use the matrix operator norm $|R|:=\max _{|v|=1}|R v|$. Since we will deal with symmetric matrices, we have the identity $|R|=\max _{|v|=1}|R v \cdot v|$.

\subsection{Beltrami flows}

We start by recalling a celebrated example of stationary periodic solutions to the 3D Euler equations, the so called Beltrami flows. One important fact which will play a central role in our paper is that the space of Beltrami flows contains linear spaces of fairly large dimension.

Proposition 3.1 (Beltrami flows) Let $\lambda_{0} \geq 1$ and let $A_{k} \in \mathbb{R}^{3}$ be such that

$$
A_{k} \cdot k=0, \quad\left|A_{k}\right|=\frac{1}{\sqrt{2}}, \quad A_{-k}=A_{k}
$$

for $k \in \mathbb{Z}^{3}$ with $|k|=\lambda_{0}$. Furthermore, let

$$
B_{k}=A_{k}+i \frac{k}{|k|} \times A_{k} \in \mathbb{C}^{3} .
$$

For any choice of $a_{k} \in \mathbb{C}$ with $\overline{a_{k}}=a_{-k}$ the vectorfield

$$
W(\xi)=\sum_{|k|=\lambda_{0}} a_{k} B_{k} e^{i k \cdot \xi}
$$

is divergence-free and satisfies

$$
\operatorname{div}(W \otimes W)=\nabla \frac{|W|^{2}}{2}
$$

Furthermore

$$
\langle W \otimes W\rangle=\int_{\mathbb{T}^{3}} W \otimes W d \xi=\frac{1}{2} \sum_{|k|=\lambda_{0}}\left|a_{k}\right|^{2}\left(\mathrm{Id}-\frac{k}{|k|} \otimes \frac{k}{|k|}\right) .
$$

In other words $W(\xi)$ defined by (19) is a stationary solution of (1) with pressure $p=-\frac{|W|^{2}}{2}$. For the rest of this paper we will treat the vectors $A_{k} \in$ $\mathbb{R}^{3}, B_{k} \in \mathbb{C}^{3}$ as fixed (the choice of $A_{k}$ as prescribed in the proposition is not unique, but this is immaterial for our purposes). The proof of Proposition 3.1 is a classic in the fluid dynamics literature, but we include it for the reader's convenience. 
Proof First of all observe that $a_{-k} B_{-k}=\overline{a_{k} B_{k}}$. Thus the vector field defined in (19) is real valued. Next notice that

$$
\operatorname{div} W(\xi)=\sum_{|k|=\lambda_{0}} i k \cdot B_{k} a_{k} e^{i k \cdot \xi}=0
$$

because $k \cdot B_{k}=0$ for every $k$.

Observe also that

$$
\operatorname{curl} W(\xi)=\sum_{|k|=\lambda_{0}} i k \times B_{k} a_{k} e^{i k \cdot \xi}
$$

On the other hand

$$
\begin{aligned}
i k \times B_{k} & =\lambda_{0}\left(i \frac{k}{|k|} \times A_{k}-\frac{k}{|k|} \times\left(\frac{k}{|k|} \times A_{k}\right)\right) \\
& =\lambda_{0}\left(i \frac{k}{|k|} \times A_{k}+A_{k}\right)=\lambda_{0} B_{k} .
\end{aligned}
$$

We therefore infer curl $W=\lambda_{0} W$. Since $W$ is divergence free, $\operatorname{div}(W \otimes W)=$ $(W \cdot \nabla) W$ and we can use the well known vector identity

$$
\operatorname{div}(W \otimes W)=(W \cdot \nabla) W=\nabla \frac{|W|^{2}}{2}-W \times(\operatorname{curl} W) .
$$

Since we have just seen that curl $W$ and $W$ are parallel, (20) follows easily.

Finally, we compute

$$
W \otimes W=\sum_{k, j} a_{k} a_{j} B_{k} \otimes B_{j} e^{i(k+j) \cdot \xi}=\sum_{k, j} a_{k} \overline{a_{j}} B_{k} \otimes \bar{B}_{j} e^{i(k-j) \cdot \xi} .
$$

Averaging this identity in $\xi$ we infer

$$
\langle W \otimes W\rangle=\sum_{|k|=\lambda_{0}}\left|a_{k}\right|^{2} B_{k} \otimes \bar{B}_{k} .
$$

However, since $B_{k}=\bar{B}_{-k}$, we get

$$
\begin{aligned}
\langle W \otimes W\rangle & =\sum_{|k|=\lambda_{0}}\left|a_{k}\right|^{2} \operatorname{Re}\left(B_{k} \otimes \bar{B}_{k}\right) \\
& =\sum_{|k|=\lambda_{0}}\left|a_{k}\right|^{2}\left(A_{k} \otimes A_{k}+\left(\frac{k}{|k|} \times A_{k}\right) \otimes\left(\frac{k}{|k|} \times A_{k}\right)\right) .
\end{aligned}
$$


On the other hand, observe that the triple $\sqrt{2} A_{k}, \sqrt{2} \frac{k}{|k|} \times A_{k}, \frac{k}{|k|}$ forms an orthonormal basis of $\mathbb{R}^{3}$. Thus,

$$
2 A_{k} \otimes A_{k}+2\left(\frac{k}{|k|} \times A_{k}\right) \otimes\left(\frac{k}{|k|} \times A_{k}\right)+\frac{k}{|k|} \otimes \frac{k}{|k|}=\mathrm{Id} .
$$

This shows (21) and hence completes the proof.

\subsection{The geometric lemma}

One key point of our construction is that the abundance of Beltrami flows allows to find several such flows $v$ with the property that

$$
\langle v \otimes v\rangle(t):=\frac{1}{(2 \pi)^{3}} \int_{\mathbb{T}^{3}} v \otimes v(x, t) d x
$$

equals a prescribed symmetric matrix $R$. Indeed we will need to select these flows so as to depend smoothly on the matrix $R$, at least when $R$ belongs to a neighborhood of the identity matrix. In view of (21), such selection is made possible by the following lemma.

Lemma 3.2 (Geometric Lemma) For every $N \in \mathbb{N}$ we can choose $r_{0}>0$ and $\lambda_{0}>1$ with the following property. There exist pairwise disjoint subsets

$$
\Lambda_{j} \subset\left\{k \in \mathbb{Z}^{3}:|k|=\lambda_{0}\right\} \quad j \in\{1, \ldots, N\}
$$

and smooth positive functions

$$
\gamma_{k}^{(j)} \in C^{\infty}\left(B_{r_{0}}(\mathrm{Id})\right) \quad j \in\{1, \ldots, N\}, k \in \Lambda_{j}
$$

such that

(a) $k \in \Lambda_{j}$ implies $-k \in \Lambda_{j}$ and $\gamma_{k}^{(j)}=\gamma_{-k}^{(j)}$;

(b) For each $R \in B_{r_{0}}(\mathrm{Id})$ we have the identity

$$
R=\frac{1}{2} \sum_{k \in \Lambda_{j}}\left(\gamma_{k}^{(j)}(R)\right)^{2}\left(\mathrm{Id}-\frac{k}{|k|} \otimes \frac{k}{|k|}\right) \quad \forall R \in B_{r_{0}}(\mathrm{Id}) .
$$

Remark 2 Though it will not be used in the sequel, the cardinality of each set $\Lambda_{j}$ constructed in the proof of the lemma is indeed bounded a priori independently of all the other parameters. A close inspection of the proof shows that it gives sets with cardinality at most 98 . This seems however far from optimal: one should be able to find sets $\Lambda_{j}$ with cardinality 14 . 
The proof of the Geometric Lemma is based on the following well-known fact.

Proposition 3.3 The set $\mathbb{Q}^{3} \cap \mathbb{S}^{2}$ is dense in $\mathbb{S}^{2}$.

Proof Let $\mathbf{s}: \mathbb{R}^{2} \rightarrow \mathbb{S}^{2}$ be the inverse of the stereographic projection:

$$
\mathbf{s}(u, v):=\left(\frac{2 v}{u^{2}+v^{2}+1}, \frac{2 u}{u^{2}+v^{2}+1}, \frac{u^{2}+v^{2}-1}{u^{2}+v^{2}+1}\right) .
$$

It is obvious that $\mathbf{s}\left(\mathbb{Q}^{2}\right) \subset \mathbb{Q}^{3}$. Since $\mathbb{Q}^{2}$ is dense in $\mathbb{R}^{2}$ and $\mathbf{s}$ is a diffeomorphism onto $\mathbb{S}^{2} \backslash(0,0,1)$, the proposition follows trivially.

Indeed, much more can be proved: $\frac{1}{n} \mathbb{Z}^{3} \cap \mathbb{S}^{2}$, distributes uniformly on the sphere for $n \in \mathbb{N}$ large whenever $n \equiv 1,2,3,4,5,6(\bmod 8)$. This problem was raised by Linnik (see [16]), who proved a first result in its direction, and solved thanks to a breakthrough of Iwaniec [14] in the theory of modular forms of half-integral weight (see, for instance, [9] and [19]).

Proof of Lemma 3.2 For each vector $v \in \mathbb{R}^{3} \backslash\{0\}$, we denote by $M_{v}$ the $3 \times 3$ symmetric matrix given by

$$
M_{v}=\mathrm{Id}-\frac{v}{|v|} \otimes \frac{v}{|v|} .
$$

With this notation the identity (22) reads as

$$
R=\frac{1}{2} \sum_{k \in \Lambda_{j}}\left(\gamma_{k}^{(j)}(R)\right)^{2} M_{k} .
$$

Step 1 Fix a $\lambda_{0}>1$ and for each set $F \subset\left\{k \in \mathbb{Z}^{3}:|k|=\lambda_{0}\right\}$ we consider the set $c(F)$ which is the interior of the convex hull, in $\mathcal{S}^{3 \times 3}$, of $\left\{M_{k}: k \in F\right\}$. We claim in this step that it suffices to find a $\lambda_{0}$ and $N$ disjoint subsets $F_{j} \subset$ $\left\{k \in \mathbb{Z}^{3}:|k|=\lambda_{0}\right\}$ such that

(d) $-F_{j}=F_{j}$;

(e) $c\left(F_{j}\right)$ contains a positive multiple of the identity.

Indeed, we will show below that, if $F_{j}$ satisfies (d) and (e), then we can find a $r_{0}>0$, a subset $\Gamma_{j} \subset F_{j}$ and positive smooth functions $\lambda_{k}^{(j)} \in C^{\infty}\left(B_{2 r_{0}}(\mathrm{Id})\right)$ such that

$$
R=\sum_{k \in \Gamma_{j}} \lambda_{k}^{(j)}(R) M_{k}
$$

We then find $\Lambda_{j}$ and the functions $\gamma_{k}^{(j)}$ by 
- defining $\Lambda_{j}:=\Gamma_{j} \cup-\Gamma_{j}$;

- setting $\lambda_{k}^{(j)}=0$ if $k \in \Lambda_{j} \backslash \Gamma_{j}$;

- defining

$$
\gamma_{k}^{(j)}:=\sqrt{\lambda_{k}^{(j)}+\lambda_{-k}^{(j)}}
$$

for every $k \in \Lambda_{j}$.

Observe that the functions and the sets satisfy both (a) and (b). Moreover, since at least one of the $\lambda_{ \pm k}^{(j)}$ is positive on $B_{2 r_{0}}(\mathrm{Id}), \gamma_{k}^{(j)}$ is smooth in $B_{r_{0}}(\mathrm{Id})$.

We now come to the existence of the set $\Gamma_{j}$. For simplicity we drop the subscripts. The open set $c(F)$ contains an element $\alpha$ Id with $\alpha>0$. Then there are seven matrices $A_{1}, \ldots, A_{7}$ in $c(F)$ such that $\alpha$ Id belongs to the interior of their convex hull, which is an open convex simplex $S$. We choose $\vartheta$ so that the ball $\tilde{U}$ of center $\alpha$ Id and radius $\vartheta$ is contained in $S$. Then each point $R \in \tilde{U}$ can be written in a unique way as a convex combination of the elements $A_{i}$ :

$$
R=\sum_{i=1}^{7} \beta_{i}(R) A_{i}
$$

and the functions $\beta_{i}$ are positive and smooth on $\tilde{U}$.

By Caratheodory's Theorem, each $A_{i}$ is the convex combination $\sum \lambda_{i, n} M_{v_{i, n}}$ of at most $7 M_{v_{i, n}}$ with $v_{i, n} \in F$, where we require that each $\lambda_{i, n}$ is positive (observe that Caratheodory's Theorem guarantees the existence of 7 points $M_{v_{i, n}}$ such that $A_{i}$ belongs to the closed convex hull of them; if we insist on the property that the corresponding coefficients are all positive, then we might be obliged to choose a number smaller than 7).

Set $r_{0}:=\frac{\vartheta}{2 \alpha}$. Then,

$$
R=\sum_{i, n} \frac{1}{\alpha} \beta_{i}(\alpha R) \lambda_{i, n} M_{v_{i, n}} \quad \forall R \in B_{2 r_{0}}(\mathrm{Id})
$$

and each coefficient

$$
\frac{1}{\alpha} \beta_{i}(\alpha R) \lambda_{i, n}
$$

is positive for every $R \in B_{2 r_{0}}(\mathrm{Id})$.

The set $\Gamma_{j}$ is then given by $\left\{v_{i, n}\right\}$. Note that we might have $v_{i, n}=v_{l, m}$ for two distinct pairs $(i, n)$ and $(l, m)$. Therefore, for $k \in \Gamma_{j}$, the function $\lambda_{k}$ will be defined as

$$
\lambda_{k}(R)=\sum_{(i, n): k=v_{i, n}} \frac{1}{\alpha} \beta_{i}(\alpha R) \lambda_{i, n} .
$$


Step 2 By Step 1, in order to prove the lemma, it suffices to find a number $\lambda_{0}$ and $N$ disjoint families $F_{1}, \ldots, F_{N} \subset \lambda_{0} \mathbb{S}^{2} \cap \mathbb{Z}^{3}$ such that the sets $c\left(F_{i}\right)$ contain all a positive multiple of the identity. By Proposition 3.3 there is a sequence $\lambda_{k} \uparrow \infty$ such that the sets $\mathbb{S}^{2} \cap \frac{1}{\lambda_{k}} \mathbb{Z}^{3}$ converge, in the Hausdorff sense, to the entire sphere $\mathbb{S}^{2}$.

Given this sequence $\left\{\lambda_{k}\right\}$, we can easily partition each $\lambda_{k} \mathbb{S}^{2} \cap \mathbb{Z}^{3}$ into $N$ disjoint symmetric families $\left\{F_{j}^{k}\right\}_{j=1, \ldots, N}$ in such a way that, for each fixed $j$, the corresponding sequence of sets $\left\{\frac{1}{\lambda_{k}} F_{j}^{k}\right\}_{k}$ converges in the Hausdorff sense to $\mathbb{S}^{2}$. Hence, any point of $c\left(\mathbb{S}^{2}\right)$ is contained in $c\left(\frac{1}{\lambda_{k}} F_{j}^{k}\right)$ provided $k$ is large enough. On the other hand it is easy to see that $c\left(\mathbb{S}^{2}\right)$ contains a multiple of the identity $\alpha$ Id (for instance one can adapt the argument of Lemma 4.2 in [6]). By Step 1, this concludes the proof.

\section{The maps $v_{1}, \stackrel{\circ}{R_{1}}$ and $p_{1}$}

We have now all the tools to define the maps $v_{1}, \stackrel{\circ}{R}_{1}$ and $p_{1}$ of Proposition 2.2. Recalling (17) and the discussion in Sect. 2.1, $w:=v_{1}-v$ is the sum of two maps, $w_{o}$ and $w_{c} . w_{o}$ is a highly oscillatory function based on "patching Beltrami flows" and it will be defined first, in Sect. 4.1. $w_{c}$ will then be added so as to ensure that $v_{1}$ is divergence free: in order to achieve this we will use the classical Leray projector, see Sect. 4.3 for the precise definition. $p_{1}$ is related to $w_{o}$ by a simple formula, given in Sect. 4.4. Finally, in Sect. 4.5 we will define $\stackrel{R}{R}_{1}$. Essentially, this last matrix field can also be thought of as a "corrector term", analogous to $w_{c}$. In fact, if we consider the point of view of [6], the Euler-Reynolds system can be stated equivalently as the fact that the $4 \times 4$ matrix

$$
U:=\left(\begin{array}{ll}
v_{1} \otimes v_{1}+p_{1} \mathrm{Id}-\stackrel{\circ}{R}_{1} & v_{1} \\
v_{1} & 0
\end{array}\right)
$$

is a divergence-free in space-time. $\stackrel{\circ}{R}_{1}$ has therefore the same flavor as $w_{c}$ and is also defined through a suitable (elliptic) operator, cf. with Definition 4.2.

\subsection{The perturbation $w_{o}$}

We start by defining a partition of unity on the space of velocities, i.e. the state space. We choose two constants $c_{1}$ and $c_{2}$ such that $\frac{\sqrt{3}}{2}<c_{1}<c_{2}<1$ and we fix a function $\varphi \in C_{c}^{\infty}\left(B_{c_{2}}(0)\right)$ which is nonnegative and identically 1 on the ball $B_{c_{1}}(0)$. We next consider the lattice $\mathbb{Z}^{3} \subset \mathbb{R}^{3}$ and its quotient by $(2 \mathbb{Z})^{3}$, i.e. we define the equivalence relation

$$
\left(k_{1}, k_{2}, k_{3}\right) \sim\left(\ell_{1}, \ell_{2}, \ell_{3}\right) \quad \Longleftrightarrow \quad k_{i}-\ell_{i} \text { is even } \forall i .
$$


We then denote by $\mathcal{C}_{j}, j=1, \ldots, 8$ the 8 equivalence classes of $\mathbb{Z}^{3} / \sim$. For each $k \in \mathbb{Z}^{3}$ denote by $\varphi_{k}$ the function

$$
\varphi_{k}(x):=\varphi(x-k) .
$$

Observe that, if $k \neq \ell \in \mathcal{C}_{i}$, then $|k-\ell| \geq 2>2 c_{2}$. Hence $\varphi_{k}$ and $\varphi_{\ell}$ have disjoint supports. On the other hand, the function

$$
\psi:=\sum_{k \in \mathbb{Z}^{3}} \varphi_{k}^{2}
$$

is smooth, bounded and bounded away from zero. We then define

$$
\alpha_{k}(v):=\frac{\varphi_{k}(v)}{\sqrt{\psi(v)}}
$$

and

$$
\phi_{k}^{(j)}(v, \tau):=\sum_{l \in \mathcal{C}_{j}} \alpha_{l}(\mu v) e^{-i\left(k \cdot \frac{l}{\mu}\right) \tau} .
$$

Since $\alpha_{l}$ and $\alpha_{\tilde{l}}$ have disjoint supports for $l \neq \tilde{l} \in \mathcal{C}_{j}$, it follows that for all $v, \tau, j$

$$
\left|\phi_{k}^{(j)}(v, \tau)\right|^{2}=\sum_{l \in \mathcal{C}_{j}} \alpha_{l}(\mu v)^{2},
$$

and in particular $\sum_{j=1}^{8}\left|\phi_{k}^{(j)}(v, \tau)\right|^{2}=1$. Furthermore, for the same reason there exist for any $m=0,1,2, \ldots$ constants $C=C(m)$ such that

$$
\sup _{v, \tau}\left|D_{v}^{m} \phi_{k}^{(j)}(v, \tau)\right| \leq C(m) \mu^{m} .
$$

Fix next any $(v, \tau)$ and $j$. Observe that there is at most one $l \in \mathcal{C}_{j}$ with the property that $\alpha_{l}(\mu v) \neq 0$ and this $l$ has the property that $|\mu v-l|<1$. Thus, in a neighborhood of $(v, \tau)$ we will have

$$
\partial_{\tau} \phi_{k}^{(j)}+i(k \cdot v) \phi_{k}^{(j)}=i k \cdot\left(v-\frac{l}{\mu}\right) \phi_{k}^{(j)} .
$$

Combining (25) and (26), for any $m=0,1,2, \ldots$ we find constants $C=$ $C(m,|k|)$ such that

$$
\sup _{v, \tau}\left|D_{v}^{m}\left(\partial_{\tau} \phi_{k}^{(j)}+i(k \cdot v) \phi_{k}^{(j)}\right)\right| \leq C(m,|k|) \mu^{m-1} .
$$

We apply Lemma 3.2 with $N=8$ to obtain $\lambda_{0}>1, r_{0}>0$ and pairwise disjoint families $\Lambda_{j}$ together with corresponding functions $\gamma_{k}^{(j)} \in C^{\infty}\left(B_{r_{0}}(\mathrm{Id})\right)$. 
Next, set

$$
\rho(t):=\frac{1}{3(2 \pi)^{3}}\left(e(t)\left(1-\frac{\delta}{2}\right)-\int_{\mathbb{T}^{3}}|v|^{2}(x, t) d x\right)
$$

and

$$
R(x, t):=\rho(t) \operatorname{Id}-\stackrel{\circ}{R}(x, t),
$$

and define

$$
w_{o}(x, t):=\sqrt{\rho(t)} \sum_{j=1}^{8} \sum_{k \in \Lambda_{j}} \gamma_{k}^{(j)}\left(\frac{R(x, t)}{\rho(t)}\right) \phi_{k}^{(j)}(v(x, t), \lambda t) B_{k} e^{i \lambda k \cdot x} .
$$

\subsection{The constants $\eta$ and $M$}

Note that $w_{o}$ is well-defined only if $\frac{R}{\rho} \in B_{r_{0}}$ (Id) where $r_{0}$ is given in Lemma 3.2. This is ensured by an appropriate choice of $\eta$. Indeed,

$$
\rho(t) \geq \frac{1}{3(2 \pi)^{3}} \frac{\delta}{4} e(t) \geq c \delta \min _{t \in[0,1]} e(t)=: c \delta m,
$$

where $c$ is a dimensional (positive) constant and $m>0$ by assumption. Then

$$
\left\|\frac{R}{\rho(t)}-\mathrm{Id}\right\| \leq \frac{1}{c \delta m}\|\stackrel{\circ}{R}\| \leq \frac{\eta}{c m} .
$$

Thus, it suffices to choose

$$
\eta:=\frac{1}{2} c m r_{0}=\frac{r_{0}}{24(2 \pi)^{3}} \min _{t \in[0,1]} e(t) .
$$

Observe that this choice is independent of $\delta>0$.

Notice next that, by our choice of $\rho(t)$ and by (7), $\rho(t) \leq \delta e(t)$. Thus there exists a constant $M>1$ depending only on $e$ (in particular independent of $\delta$ ) so that

$$
\sup _{t \in[0,1]} \sup _{x \in \mathbb{T}^{3}}\left|w_{o}(x, t)\right| \leq \frac{\sqrt{M \delta}}{2} .
$$

This fixes the choice of the constant $M$ in Proposition 2.2.

\subsection{The correction $w_{c}$}

We next define the Leray projector onto divergence-free vectorfields with zero average. 
Definition 4.1 Let $v \in C^{\infty}\left(\mathbb{T}^{3}, \mathbb{R}^{3}\right)$ be a smooth vector field. Let

$$
\mathcal{Q} v:=\nabla \phi+\int_{\mathbb{T}^{3}} v,
$$

where $\phi \in C^{\infty}\left(\mathbb{T}^{3}\right)$ is the solution of

$$
\Delta \phi=\operatorname{div} v \text { in } \mathbb{T}^{3}
$$

with $\int_{\mathbb{T}^{3}} \phi=0$. Furthermore, let $\mathcal{P}=I-\mathcal{Q}$ be the Leray projection onto divergence-free fields with zero average.

The vector field $v_{1}$ is then the sum of $v$ with the Leray projection $w$ of $w_{o}$, namely

$$
v_{1}(x, t):=v(x, t)+\mathcal{P} w_{o}(x, t)=: v(x, t)+w(x, t)
$$

and hence

$$
w_{c}(x, t):=-\mathcal{Q} w_{o}(x, t)=w(x, t)-w_{o}(x, t) .
$$

\subsection{The pressure $p_{1}$}

We define

$$
p_{1}:=p-\frac{\left|w_{o}\right|^{2}}{2}
$$

4.5 The Reynolds stress $\stackrel{\circ}{R}_{1}$

In order to specify the choice of $\stackrel{\circ}{R}_{1}$ we introduce a new operator.

Definition 4.2 Let $v \in C^{\infty}\left(\mathbb{T}^{3}, \mathbb{R}^{3}\right)$ be a smooth vector field. We then define $\mathcal{R} v$ to be the matrix-valued periodic function

$$
\mathcal{R} v:=\frac{1}{4}\left(\nabla \mathcal{P} u+(\nabla \mathcal{P} u)^{T}\right)+\frac{3}{4}\left(\nabla u+(\nabla u)^{T}\right)-\frac{1}{2}(\operatorname{div} u) \operatorname{Id},
$$

where $u \in C^{\infty}\left(\mathbb{T}^{3}, \mathbb{R}^{3}\right)$ is the solution of

$$
\Delta u=v-\oint_{\mathbb{T}^{3}} v \text { in } \mathbb{T}^{3}
$$

with $\oint_{\mathbb{T}^{3}} u=0$.

Lemma $4.3\left(\mathcal{R}=\operatorname{div}^{-1}\right)$ For any $v \in C^{\infty}\left(\mathbb{T}^{3}, \mathbb{R}^{3}\right)$ we have

(a) $\mathcal{R} v(x)$ is a symmetric trace-free matrix for each $x \in \mathbb{T}^{3}$;

(b) $\operatorname{div} \mathcal{R} v=v-\oint_{\mathbb{T}^{3}} v$. 
Proof It is obvious by inspection that $\mathcal{R} v$ is symmetric. Since $\mathcal{P} v$ is divergence-free, we obtain for the trace

$$
\operatorname{tr}(\mathcal{R} v)=\frac{3}{4}(2 \operatorname{div} u)-\frac{3}{2} \operatorname{div} u=0 .
$$

Similarly, we have

$$
\operatorname{div}(\mathcal{R} v)=\frac{1}{4} \Delta(\mathcal{P} u)+\frac{3}{4}(\nabla \operatorname{div} u+\Delta u)-\frac{1}{2} \nabla \operatorname{div} u .
$$

On the other hand recall that $\mathcal{P} u=u-\nabla \phi-\{u=u-\nabla \phi$, where $\Delta \phi=$ $\operatorname{div} u$. Therefore $\Delta(\mathcal{P} u)=\Delta u-\nabla \operatorname{div} u$. Plugging this identity into (32), we obtain

$$
\operatorname{div}(\mathcal{R} v)=\Delta u
$$

and since $u$ solves $\Delta u=v-\oint v$, (b) follows readily.

Then we set

$$
\stackrel{\circ}{R}_{1}:=\mathcal{R}\left(\partial_{t} v_{1}+\operatorname{div}\left(v_{1} \otimes v_{1}\right)+\nabla p_{1}\right) .
$$

Note that $\left[\partial_{t} v_{1}+\operatorname{div}\left(v_{1} \otimes v_{1}\right)+\nabla p_{1}\right]$ has average zero. Indeed:

- the vector field $\operatorname{div}\left(v_{1} \otimes v_{1}\right)+\nabla p_{1}$ has average 0 because it is the divergence of the matrix field $v_{1} \otimes v_{1}+p_{1} \mathrm{Id}$;

- for the same reason, the identity $\partial_{t} v=-\operatorname{div}(v \otimes v+p \operatorname{Id}-\stackrel{R}{R})$ shows that $\partial_{t} v$ has average 0 ; on the other hand $w=\mathcal{P} w_{o}$ has average 0 because of the definition of $\mathcal{P}$; this implies that $\partial_{t} w$ has also average zero and thus we conclude as well that $\partial_{t} v_{1}=\partial_{t} v+\partial_{t} w$ has average zero.

Therefore from Lemma 4.3 it follows that $\stackrel{\circ}{R}_{1}(x, t)$ is symmetric and tracefree and that the identity

$$
\partial_{t} v_{1}+\operatorname{div}\left(v_{1} \otimes v_{1}\right)+\nabla p_{1}=\operatorname{div} \stackrel{\circ}{R}_{1}
$$

holds.

The rest of this note is devoted to prove that the triple $\left(v_{1}, p_{1}, \stackrel{\circ}{R}_{1}\right)$ satisfies the estimates (9), (10), (11) and (12). This will be achieved by an appropriate choice of the parameters $\mu$ and $\lambda$. In particular, we will show that the estimates hold provided $\mu$ is sufficiently large and $\lambda$ much larger than $\mu$.

\section{Schauder estimates}

In the following $m=0,1,2, \ldots, \alpha \in(0,1)$, and $\beta$ is a multiindex. We introduce the usual (spatial) Hölder norms as follows. First of all, the supremum 
norm is denoted by $\|f\|_{0}:=\sup _{\mathbb{T}^{3}}|f|$. We define the Hölder seminorms as

$$
\begin{aligned}
{[f]_{m} } & =\max _{|\beta|=m}\left\|D^{\beta} f\right\|_{0}, \\
{[f]_{m+\alpha} } & =\max _{|\beta|=m} \sup _{x \neq y} \frac{\left|D^{\beta} f(x)-D^{\beta} f(y)\right|}{|x-y|^{\alpha}} .
\end{aligned}
$$

The Hölder norms are then given by

$$
\begin{aligned}
\|f\|_{m} & =\sum_{j=0}^{m}[f]_{j} \\
\|f\|_{m+\alpha} & =\|f\|_{m}+[f]_{m+\alpha} .
\end{aligned}
$$

Recall the following elementary inequalities:

$$
[f]_{s} \leq C\left(\varepsilon^{r-s}[f]_{r}+\varepsilon^{-s}\|f\|_{0}\right)
$$

for $r \geq s \geq 0$ and all $\varepsilon>0$, and

$$
\left.[f g]_{r} \leq C([f]]_{r}\|g\|_{0}+\|f\|_{0}[g]_{r}\right)
$$

for any $1 \geq r \geq 0$.

Finally, we recall the classical Schauder estimates for the Laplace operator and the corresponding estimates which we can infer for the various operators involved in our construction.

Proposition 5.1 For any $\alpha \in(0,1)$ and any $m \in \mathbb{N}$ there exists a constant $C(\alpha, m)$ with the following properties. If $\phi, \psi: \mathbb{T}^{3} \rightarrow \mathbb{R}$ are the unique solutions of

$$
\left\{\begin{array} { l } 
{ \Delta \phi = f , } \\
{ f \phi = 0 , }
\end{array} \quad \left\{\begin{array}{l}
\Delta \psi=\operatorname{div} F, \\
f \psi=0,
\end{array}\right.\right.
$$

then

$$
\|\phi\|_{m+2+\alpha} \leq C(m, \alpha)\|f\|_{m, \alpha} \quad \text { and } \quad\|\psi\|_{m+1+\alpha} \leq C(m, \alpha)\|F\|_{m, \alpha} .
$$

Moreover we have the estimates

$$
\begin{aligned}
\|\mathcal{Q} v\|_{m+\alpha} & \leq C(m, \alpha)\|v\|_{m+\alpha} \\
\|\mathcal{P} v\|_{m+\alpha} & \leq C(m, \alpha)\|v\|_{m+\alpha} \\
\|\mathcal{R} v\|_{m+1+\alpha} & \leq C(m, \alpha)\|v\|_{m+\alpha} \\
\|\mathcal{R}(\operatorname{div} A)\|_{m+\alpha} & \leq C(m, \alpha)\|A\|_{m+\alpha}
\end{aligned}
$$




$$
\|\mathcal{R} \mathcal{Q}(\operatorname{div} A)\|_{m+\alpha} \leq C(m, \alpha)\|A\|_{m+\alpha} .
$$

Proof The estimates (35) are the usual Schauder estimates, see for instance [12, Chap. 4]. The meticulous reader will notice that the estimates in [12] are stated in $\mathbb{R}^{n}$ for the potential-theoretic solution of the Laplace operator. The periodic case is however an easy corollary. Take for instance $\phi$ and $f$ and consider them as periodic functions defined on $\mathbb{R}^{3}$. Consider $g=f \chi$, where $\chi$ is a cut-off function supported in $B_{6 \pi}(0)$ and identically 1 on $B_{4 \pi}(0)$. Let $\tilde{\phi}$ be the potential-theoretic solution in $\mathbb{R}^{3}$ of $\Delta \tilde{\phi}=g$. For $\tilde{\phi}$ we can invoke the Schauder estimates as in [12, Chap. 4]. Moreover $\phi-\tilde{\phi}$ is an harmonic function in $B_{4 \pi}(0)$. Obviously $\|\phi\|_{L^{2}\left(B_{4 \pi}(0)\right)}$ can be easily bounded using $\Delta \phi=f, \oint \phi=0$ and the Parseval identity. Thus, standard properties of harmonic functions give $\|\phi-\tilde{\phi}\|_{C^{m, \alpha}\left([2 \pi]^{3}\right)} \leq C(m, \alpha)\|f\|_{0}$.

The estimates (36), (37), (38) and (39) are easy consequences of (35) and the definitions of the operators. The estimate (40) requires a little more care. Let $u: \mathbb{T}^{3} \rightarrow \mathbb{R}^{3}$ be the unique solution of

$$
\Delta \Delta u_{i}=\partial_{i} \sum_{j, n} \partial_{j n}^{2} A_{j n}
$$

with $f u=0$. Then

$$
\|u\|_{m+1+\alpha} \leq C(m, \alpha)\|A\|_{m+\alpha} .
$$

First of all, with the argument above, one can reduce this estimate to a corresponding one for the potential-theoretic solution of the biLaplace operator in $\mathbb{R}^{3}$. For this case we can then invoke general estimates for elliptic $k$-homogeneous constant coefficients operators (see for instance [24, Theorem 1]) or use the same arguments of [12, Chap. 4] replacing the fundamental solution of the Laplacian with that of the biLaplacian. Finally, (40) follows from the identity

$$
\mathcal{R Q}(\operatorname{div} A)=\frac{1}{4}\left(\nabla \mathcal{P} u+(\nabla \mathcal{P} u)^{T}\right)+\frac{3}{4}\left(\nabla u+(\nabla u)^{T}\right)-\frac{1}{2}(\operatorname{div} u) \operatorname{Id}
$$

and the estimates (41) and (37).

In what follows we will use the convention that Greek subscripts of Hölder norms denote always exponents in the open interval $(0,1)$.

Proposition 5.2 Let $k \in \mathbb{Z}^{3} \backslash\{0\}$ and $\lambda \geq 1$ be fixed.

(i) For any $a \in C^{\infty}\left(\mathbb{T}^{3}\right)$ and $m \in \mathbb{N}$ we have

$$
\left|\int_{\mathbb{T}^{3}} a(x) e^{i \lambda k \cdot x} d x\right| \leq C \frac{[a]_{m}}{\lambda^{m}} .
$$


(ii) Let $\lambda \in \mathbb{N} \backslash\{0\}$ and $\phi_{\lambda} \in C^{\infty}\left(\mathbb{T}^{3}\right)$ be the solution of

$$
\Delta \phi_{\lambda}=f_{\lambda} \text { in } \mathbb{T}^{3}
$$

with $\int_{\mathbb{T}^{3}} \phi_{\lambda}=0$, where

$$
f_{\lambda}(x):=a(x) e^{i \lambda k \cdot x}-\oint_{\mathbb{T}^{3}} a(y) e^{i \lambda k \cdot y} d y .
$$

Then for any $\alpha \in(0,1)$ and $m \in \mathbb{N}$ we have the estimate

$$
\left\|\nabla \phi_{\lambda}\right\|_{\alpha} \leq \frac{C}{\lambda^{1-\alpha}}\|a\|_{0}+\frac{C}{\lambda^{m-\alpha}}[a]_{m}+\frac{C}{\lambda^{m}}[a]_{m+\alpha},
$$

where $C=C(\alpha, m)$.

Proof For $j=0,1, \ldots$ define

$$
\begin{aligned}
& A_{j}(y, \xi):=-i\left[\frac{k}{|k|^{2}}\left(i \frac{k}{|k|^{2}} \cdot \nabla\right)^{j} a(y)\right] e^{i k \cdot \xi}, \\
& F_{j}(y, \xi):=\left[\left(i \frac{k}{|k|^{2}} \cdot \nabla\right)^{j} a(y)\right] e^{i k \cdot \xi} .
\end{aligned}
$$

Direct calculation shows that

$$
F_{j}(x, \lambda x)=\frac{1}{\lambda} \operatorname{div}\left[A_{j}(x, \lambda x)\right]+\frac{1}{\lambda} F_{j+1}(x, \lambda x) .
$$

In particular for any $m \in \mathbb{N}$

$$
a(x) e^{i \lambda k \cdot x}=F_{0}(x, \lambda x)=\frac{1}{\lambda} \sum_{j=0}^{m-1} \frac{1}{\lambda^{j}} \operatorname{div}\left[A_{j}(x, \lambda x)\right]+\frac{1}{\lambda^{m}} F_{m}(x, \lambda x) .
$$

Integrating this over $\mathbb{T}^{3}$ and using that $|k| \geq 1$ we obtain (42).

Next, using (33) and (34) we have, for any $j \leq m-1$,

$$
\begin{aligned}
\left\|A_{j}(\cdot, \lambda \cdot)\right\|_{\alpha} & \leq C\left(\lambda^{\alpha}[a]_{j}+[a]_{j+\alpha}\right) \\
& \leq C \lambda^{j+\alpha}\left(\lambda^{-m}[a]_{m}+\|a\|_{0}\right)
\end{aligned}
$$

and similarly

$$
\left\|F_{m}(\cdot, \lambda \cdot)\right\|_{\alpha} \leq C\left(\lambda^{\alpha}[a]_{m}+[a]_{m+\alpha}\right) .
$$


Moreover, according to the standard estimate (35),

$$
\begin{aligned}
\|\nabla \phi\|_{\alpha} \leq & C\left(\frac{1}{\lambda} \sum_{j=0}^{m-1} \frac{1}{\lambda^{j}}\left\|A_{j}(\cdot, \lambda \cdot)\right\|_{\alpha}+\frac{1}{\lambda^{m}}\left\|F_{m}(\cdot, \lambda \cdot)\right\|_{\alpha}\right. \\
& \left.+\left|\oint_{\mathbb{T}^{3}} F_{0}(x, \lambda x) d x\right|\right),
\end{aligned}
$$

hence, using (42) for the last term,

$$
\|\nabla \phi\|_{\alpha} \leq \frac{C}{\lambda^{1-\alpha}}\|a\|_{0}+\frac{C}{\lambda^{m-\alpha}}[a]_{m}+\frac{C}{\lambda^{m}}[a]_{m+\alpha}
$$

as required.

Corollary 5.3 Let $k \in \mathbb{Z}^{3} \backslash\{0\}$ be fixed. For a smooth vectorfield a $\in$ $C^{\infty}\left(\mathbb{T}^{3} ; \mathbb{R}^{3}\right)$ let $F(x):=a(x) e^{i \lambda k \cdot x}$. Then we have

$$
\|\mathcal{R}(F)\|_{\alpha} \leq \frac{C}{\lambda^{1-\alpha}}\|a\|_{0}+\frac{C}{\lambda^{m-\alpha}}[a]_{m}+\frac{C}{\lambda^{m}}[a]_{m+\alpha},
$$

where $C=C(\alpha, m)$.

Proof This is an immediate consequence of the definition of $\mathcal{R}$, the Schauder estimate (37) for $\mathcal{P}$ and Proposition 5.2 above.

\section{Estimates on the corrector and the energy}

In all subsequent estimates, unless otherwise stated, $C$ denotes a generic constant that can vary from line to line, and depends on $e, v, \stackrel{R}{R}$ as well as on $\lambda_{0}, \alpha$ and $\delta$, but is independent of $\lambda$ and $\mu$. Smallness of the respective quantities will be achieved by an appropriate choice of $\lambda, \mu$ in Sect. 8. Moreover, all estimates will implicitly assume (18), in particular that $1 \leq \mu \leq \lambda$.

Our aim is to estimate the space-time sup-norm of $v_{1}-v=w=w_{o}+w_{c}$ and $\stackrel{R}{R}_{1}$. Since $w_{c}$ and $\stackrel{\circ}{R}_{1}$ are defined in terms of the singular integral operators $\mathcal{P}, \mathcal{Q}$ and $\mathcal{R}$, which act in space, instead of obtaining directly estimates of the $C^{0}$ norm, we will use Schauder estimates to obtain bounds on spatial Hölder norms. Thus, in the sequel the Hölder norms will denote spatial norms, and are understood to be uniform in time $t \in[0,1]$. Moreover, if the Hölder exponent is denoted by a Greek letter, then it is a number in the open interval $(0,1)$.

It will be convenient to write $w_{o}$ as

$$
w_{o}(x, t)=W(x, t, \lambda t, \lambda x),
$$


where

$$
\begin{aligned}
W(y, s, \tau, \xi) & :=\sum_{|k|=\lambda_{0}} a_{k}(y, s, \tau) B_{k} e^{i k \cdot \xi} \\
& =\sqrt{\rho(s)} \sum_{j=1}^{8} \sum_{k \in \Lambda_{j}} \gamma_{k}^{(j)}\left(\frac{R(y, s)}{\rho(s)}\right) \phi_{k}^{(j)}(v(y, s), \tau) B_{k} e^{i k \cdot \xi} .
\end{aligned}
$$

We summarize the main properties of the coefficients $W$ :

Proposition 6.1 (i) Let $a_{k} \in C^{\infty}\left(\mathbb{T}^{3} \times[0,1] \times \mathbb{R}\right)$ be given by (44). Then for any $r \geq 0$

$$
\begin{array}{r}
\left\|a_{k}(\cdot, s, \tau)\right\|_{r} \leq C \mu^{r}, \\
\left\|\partial_{s} a_{k}(\cdot, s, \tau)\right\|_{r} \leq C \mu^{r+1}, \\
\left\|\partial_{\tau} a_{k}(\cdot, s, \tau)\right\|_{r} \leq C \mu^{r}, \\
\left\|\left(\partial_{\tau} a_{k}+i(k \cdot v) a_{k}\right)(\cdot, s, \tau)\right\|_{r} \leq C \mu^{r-1} .
\end{array}
$$

(ii) The matrix-function $W \otimes W$ can be written as

$$
(W \otimes W)(y, s, \tau, \xi)=R(y, s)+\sum_{1 \leq|k| \leq 2 \lambda_{0}} U_{k}(y, s, \tau) e^{i k \cdot \xi}
$$

where the coefficients $U_{k} \in C^{\infty}\left(\mathbb{T}^{3} \times[0,1] \times \mathbb{R} ; \mathcal{S}^{3 \times 3}\right)$ satisfy

$$
U_{k} k=\frac{1}{2}\left(\operatorname{tr} U_{k}\right) k
$$

and for any $r \geq 0$

$$
\begin{aligned}
\left\|U_{k}^{\mu}(\cdot, s, \tau)\right\|_{r} & \leq C \mu^{r}, \\
\left\|\partial_{s} U_{k}^{\mu}(\cdot, s, \tau)\right\|_{r} & \leq C \mu^{r+1}, \\
\left\|\partial_{\tau} U_{k}(\cdot, s, \tau)\right\|_{r} & \leq C \mu^{r}, \\
\left\|\left(\partial_{\tau} U_{k}^{\mu}+i(k \cdot v) U_{k}^{\mu}\right)(\cdot, s, \tau)\right\|_{r} & \leq C \mu^{r-1} .
\end{aligned}
$$

In all these estimates the constant $C$ depends on $r$ and $e, v, \stackrel{\circ}{R}$ but is independent of $(s, \tau)$ and $\mu$.

Proof The estimates for $a_{k}$ are a consequence of (25) and (27). Indeed, since the constants in the estimates are allowed to depend on $e, v, \stackrel{\circ}{R}$, one only needs 
to keep track of the number of derivatives of $\phi_{k}^{(j)}$ with respect to $v$. Then the estimates on $a_{k}, \partial_{s} a_{k}$ and $\partial_{\tau} a_{k}+i(k \cdot v) a_{k}$ immediately follow. From the triangle inequality we can then also conclude the estimate on $\partial_{\tau} a_{k}$.

Next, consider the expansion of $\xi \mapsto W \otimes W$ into a Fourier series in $\xi$, i.e.

$$
(W \otimes W)(y, s, \tau, \xi)=U_{0}(y, s, \tau)+\sum_{1 \leq|k| \leq 2 \lambda_{0}} U_{k}(y, s, \tau) e^{i k \cdot \xi} .
$$

Since each $U_{k}$ is the sum of finitely many terms of the form $a_{k^{\prime}} a_{k^{\prime \prime}}$, the estimates for $U_{k}$ follow from those for $a_{k}$.

Next, since $U_{0}$ is given by the average (in $\xi$ ), in order to obtain (45) we need to show that

$$
\oint_{\mathbb{T}^{3}} W \otimes W(y, s, \tau, \xi) d \xi=R(y, s) .
$$

To this end we calculate:

$$
\begin{aligned}
\int_{\mathbb{T}^{3}} W \otimes W d \xi & \stackrel{(21)}{=} \frac{\rho}{2} \sum_{j} \sum_{k \in \Lambda_{j}}\left(\gamma_{k}^{(j)}\left(\rho^{-1} R\right)\right)^{2}\left|\phi_{k}^{(j)}(v, \tau)\right|^{2}\left(\operatorname{Id}-\frac{k}{|k|} \otimes \frac{k}{|k|}\right) \\
& \stackrel{(24)}{=} \frac{\rho}{2} \sum_{j} \sum_{k \in \Lambda_{j}} \sum_{l \in \mathcal{C}_{j}}\left(\gamma_{k}^{(j)}\left(\rho^{-1} R\right)\right)^{2}\left(\alpha_{l}(\mu v)\right)^{2}\left(\operatorname{Id}-\frac{k}{|k|} \otimes \frac{k}{|k|}\right) \\
& \stackrel{(22)}{=} R \sum_{j} \sum_{l \in \mathcal{C}_{j}}\left(\alpha_{l}(\mu v)\right)^{2}=R \sum_{l \in \mathbb{Z}^{3}}\left(\alpha_{l}(\mu v)\right)^{2}=R .
\end{aligned}
$$

Finally, (46) is a direct consequence of Proposition 3.1, in particular of (20).

After this preparation we are ready to estimate all the terms in the perturbation scheme. First of all we verify that the corrector term $w_{c}$ is indeed much smaller than the main perturbation term $w_{o}$ :

Lemma 6.2 (Estimate on the corrector)

$$
\left\|w_{c}\right\|_{\alpha} \leq C \frac{\mu}{\lambda^{1-\alpha}}
$$

Proof We start with the observation that, since $k \cdot B_{k}=0$,

$$
\begin{aligned}
w_{o}(x, t)= & \frac{1}{\lambda} \nabla \times\left(\sum_{|k|=\lambda_{0}}-i a_{k}(x, t, \lambda t) \frac{k \times B_{k}}{|k|^{2}} e^{i \lambda x \cdot k}\right) \\
& +\frac{1}{\lambda} \sum_{|k|=\lambda_{0}} i \nabla a_{k}(x, t, \lambda t) \times \frac{k \times B_{k}}{|k|^{2}} e^{i \lambda x \cdot k} .
\end{aligned}
$$


Hence

$$
w_{c}(x, t)=-\frac{1}{\lambda} \mathcal{Q} u_{c}(x, t),
$$

where

$$
u_{c}(x, t)=\sum_{|k|=\lambda_{0}} i \nabla a_{k}(x, t, \lambda t) \times \frac{k \times B_{k}}{|k|^{2}} e^{i \lambda x \cdot k} .
$$

The estimate (47) then follows from the Schauder estimate (36) for $\mathcal{Q}$ combined with

$$
\left\|u_{c}\right\|_{\alpha} \leq C \mu \lambda^{\alpha} .
$$

Next, we verify the estimate on the energy (9).

Lemma 6.3 (Estimate on the energy)

$$
\left.\left|e(t)\left(1-\frac{1}{2} \delta\right)-\int_{\mathbb{T}^{3}}\right| v_{1}\right|^{2} d x \mid \leq C \frac{\mu}{\lambda^{1-\alpha}} .
$$

Proof Taking the trace of identity (45) in Proposition 6.1 we have

$$
|W(y, s, \tau, \xi)|^{2}=\operatorname{tr} R(y, s)+\sum_{1 \leq|k| \leq 2 \lambda_{0}} c_{k}(y, s, \tau) e^{i k \cdot \xi}
$$

for some coefficients $c_{k} \in C^{\infty}\left(\mathbb{T}^{3} \times[0,1] \times \mathbb{R}\right)$, which satisfy the estimates

$$
\left\|c_{k}(\cdot, s, \tau)\right\|_{r} \leq C \mu^{r} .
$$

From part (i) of Proposition 5.2 with $m=1$ we deduce

$$
\left.\left|\int_{\mathbb{T}^{3}}\right| w_{o}\right|^{2}-\operatorname{tr} R d x \mid \leq C \frac{\mu}{\lambda}
$$

and

$$
\left|\int_{\mathbb{T}^{3}} v \cdot w_{o} d x\right| \leq C \frac{\mu}{\lambda} .
$$

Hence, combining with (47) we see that

$$
\left.\left|\int_{\mathbb{T}^{3}}\right| v_{1}\right|^{2}-|v|^{2}-\left|w_{o}\right|^{2} d x \mid \leq C \frac{\mu}{\lambda^{1-\alpha}} .
$$

Recalling that

$$
\operatorname{tr} R=3 \rho=\frac{1}{(2 \pi)^{3}}\left(e(t)\left(1-\frac{1}{2} \delta\right)-\int_{\mathbb{T}^{3}}|v|^{2} d x\right),
$$

we conclude (49). 


\section{Estimates on the Reynolds stress}

Rewrite

$$
\begin{aligned}
\partial_{t} v_{1} & +\operatorname{div}\left(v_{1} \otimes v_{1}\right)+\nabla p_{1} \\
= & {\left[\partial_{t} w_{o}+v \cdot \nabla w_{o}\right]+\left[\operatorname{div}\left(w_{o} \otimes w_{o}-\frac{1}{2}\left|w_{o}\right|^{2} \mathrm{Id}+\stackrel{\circ}{R}\right)\right] } \\
& +\left[\partial_{t} w_{c}+\operatorname{div}\left(v_{1} \otimes w_{c}+w_{c} \otimes v_{1}-w_{c} \otimes w_{c}+v \otimes w_{o}\right)\right] .
\end{aligned}
$$

In other words we split the Reynolds stress into the three parts on the right hand side. We will refer to them as the transport part, the oscillation part, and the error. In the following we will estimate each term separately.

Lemma 7.1 (The transport part)

$$
\left\|\mathcal{R}\left(\partial_{t} w_{o}+v \cdot \nabla w_{o}\right)\right\|_{\alpha} \leq C\left(\frac{\lambda^{\alpha}}{\mu}+\frac{\mu^{2}}{\lambda^{1-\alpha}}\right) .
$$

Proof Observe that

$$
\begin{aligned}
\mathcal{R}\left(\partial_{t} w_{o}+v \cdot \nabla w_{o}\right)= & \lambda \mathcal{R}\left(\sum_{|k|=\lambda_{0}}\left(\partial_{\tau} a_{k}+i(k \cdot v) a_{k}\right)(x, t, \lambda t) B_{k} e^{i \lambda k \cdot x}\right) \\
& +\mathcal{R}\left(\sum_{|k|=\lambda_{0}}\left(\partial_{s} a_{k}+v \cdot \nabla_{y} a_{k}\right)(x, t, \lambda t) B_{k} e^{i \lambda k \cdot x}\right) .
\end{aligned}
$$

For the first term Corollary 5.3 with $m=2$ implies the bound

$$
\frac{\lambda^{\alpha}}{\mu}+\frac{\mu}{\lambda^{1-\alpha}}+\frac{\mu^{1+\alpha}}{\lambda}
$$

whereas for the second term Corollary 5.3 with $m=1$ implies the bound

$$
\frac{\mu}{\lambda^{1-\alpha}}+\frac{\mu^{2}}{\lambda^{1-\alpha}}+\frac{\mu^{2+\alpha}}{\lambda} .
$$

Since $1 \leq \mu \leq \lambda$, we obtain (51).

Lemma 7.2 (The oscillation part)

$$
\left\|\mathcal{R}\left(\operatorname{div}\left(w_{o} \otimes w_{o}-\frac{1}{2}\left|w_{o}\right|^{2} \operatorname{Id}+\stackrel{\circ}{R}\right)\right)\right\|_{\alpha} \leq C \frac{\mu^{2}}{\lambda^{1-\alpha}} .
$$


Proof Recall the formula (45) from Proposition 6.1. Since $\rho$ is a function of $t$ only, we can write the oscillation part in (50) as

$$
\begin{aligned}
& \operatorname{div}\left(w_{o} \otimes w_{o}-\frac{1}{2}\left(\left|w_{o}\right|^{2}-\rho\right) \operatorname{Id}+\stackrel{\circ}{R}\right) \\
& =\operatorname{div}\left(w_{o} \otimes w_{o}-R-\frac{1}{2}\left(\left|w_{o}\right|^{2}-\operatorname{tr} R\right) \operatorname{Id}\right) \\
& =\operatorname{div}\left[\sum_{1 \leq|k| \leq 2 \lambda_{0}}\left(U_{k}-\frac{1}{2}\left(\operatorname{tr} U_{k}\right) \operatorname{Id}\right)(x, t, \lambda t) e^{i \lambda k \cdot x}\right] \\
& \stackrel{(46)}{=} \sum_{1 \leq|k| \leq 2 \lambda_{0}} \operatorname{div}_{y}\left[U_{k}-\frac{1}{2}\left(\operatorname{tr} U_{k}\right) \operatorname{Id}\right](x, t, \lambda t) e^{i \lambda k \cdot x} .
\end{aligned}
$$

Corollary 5.3 with $m=1$ then implies (52).

Concerning the error, we are going to treat three terms separately, as follows.

Lemma 7.3 (Estimate on the error I)

$$
\left\|\mathcal{R}\left(\partial_{t} w_{c}\right)\right\|_{\alpha} \leq C \frac{\mu^{2}}{\lambda^{1-\alpha}} .
$$

Proof Recall from (48) that $w_{c}=-\frac{1}{\lambda} \mathcal{Q} u_{c}$. Now

$$
\begin{aligned}
\partial_{t} u_{c}(x, t)= & \lambda \sum_{|k|=\lambda_{0}} i\left(\nabla \partial_{\tau} a_{k}\right)(x, t, \lambda t) \times \frac{k \times B_{k}}{|k|^{2}} e^{i \lambda x \cdot k} \\
& +\sum_{|k|=\lambda_{0}} i\left(\nabla \partial_{s} a_{k}\right)(x, t, \lambda t) \times \frac{k \times B_{k}}{|k|^{2}} e^{i \lambda x \cdot k} .
\end{aligned}
$$

Moreover, for any $c_{k} \in C^{\infty}\left(\mathbb{T}^{3} ; \mathbb{R}^{3}\right)$ we have

$$
\begin{aligned}
c_{k}(x, t, \lambda t) e^{i \lambda x \cdot k}= & \frac{1}{i \lambda} \operatorname{div}\left[c_{k}(x, t, \lambda t) \otimes \frac{k}{|k|^{2}} e^{i \lambda x \cdot k}\right] \\
& -\frac{1}{i \lambda}\left[\left(\frac{k}{|k|^{2}} \cdot \nabla\right) c_{k}(x, t, \lambda t)\right] e^{i \lambda x \cdot k} .
\end{aligned}
$$

Therefore $\partial_{t} u_{c}$ can be written as

$$
\partial_{t} u_{c}=\operatorname{div} U_{c}+\tilde{u}_{c},
$$


where

$$
\left\|U_{c}\right\|_{\alpha} \leq C \mu \lambda^{\alpha}, \quad\left\|\tilde{u}_{c}\right\|_{\alpha} \leq C \mu^{2} \lambda^{\alpha} .
$$

Therefore we have $\mathcal{R} \partial_{t} w_{c}=-\frac{1}{\lambda}\left(\mathcal{R} \mathcal{Q} \operatorname{div} U_{c}+\mathcal{R} \mathcal{Q} \tilde{u}_{c}\right)$. From the Schauder estimate (40) for the operator $\mathcal{R} \mathcal{Q} \operatorname{div}$, we conclude that

$$
\begin{aligned}
\left\|\mathcal{R} \partial_{t} w_{c}\right\|_{\alpha} & \leq \frac{1}{\lambda}\left(\left\|\mathcal{R} \mathcal{Q} \operatorname{div} U_{c}\right\|_{\alpha}+\left\|\mathcal{R} \mathcal{Q} \tilde{u}_{c}\right\|_{\alpha}\right) \\
& \leq \frac{C}{\lambda}\left(\left\|U_{c}\right\|_{\alpha}+\left\|\tilde{u}_{c}\right\|_{\alpha}\right) \leq C \frac{\mu^{2}}{\lambda^{1-\alpha}} .
\end{aligned}
$$

Lemma 7.4 (Estimate on the error II)

$$
\left\|\mathcal{R}\left(\operatorname{div}\left(v_{1} \otimes w_{c}+w_{c} \otimes v_{1}-w_{c} \otimes w_{c}\right)\right)\right\|_{\alpha} \leq C \frac{\mu}{\lambda^{1-2 \alpha}} .
$$

Proof We first estimate

$$
\begin{aligned}
& \left\|v_{1} \otimes w_{c}+w_{c} \otimes v_{1}-w_{c} \otimes w_{c}\right\|_{\alpha} \\
& \quad \leq C\left(\left\|v_{1}\right\|_{0}\left\|w_{c}\right\|_{\alpha}+\left\|v_{1}\right\|_{\alpha}\left\|w_{c}\right\|_{0}+\left\|w_{c}\right\|_{0}\left\|w_{c}\right\|_{\alpha}\right) \\
& \quad \stackrel{(47)}{\leq} C \frac{\mu}{\lambda^{1-\alpha}}\left(\left\|v_{1}\right\|_{\alpha}+\left\|w_{c}\right\|_{\alpha}\right) \\
& \quad \leq C \frac{\mu}{\lambda^{1-\alpha}}\left(\|v\|_{\alpha}+\left\|w_{c}\right\|_{\alpha}+\left\|w_{o}\right\|_{\alpha}\right) \\
& \quad \leq C \frac{\mu}{\lambda^{1-\alpha}}\left(C+C \frac{\mu}{\lambda^{1-\alpha}}+C \lambda^{\alpha}\right) .
\end{aligned}
$$

Recall that $1 \leq \mu \leq \lambda$ and hence $1 \leq \frac{\mu}{\lambda^{1-\alpha}} \leq \lambda^{\alpha}$. Thus we conclude

$$
\left\|v_{1} \otimes w_{c}+w_{c} \otimes v_{1}-w_{c} \otimes w_{c}\right\|_{\alpha} \leq C \frac{\mu}{\lambda^{1-2 \alpha}}
$$

(54) follows from the latter inequality and the Schauder estimate (39).

Lemma 7.5 (Estimate on the error III)

$$
\left\|\mathcal{R}\left(\operatorname{div}\left(v \otimes w_{o}\right)\right)\right\|_{\alpha} \leq C \frac{\mu^{2}}{\lambda^{1-\alpha}} .
$$

Proof Since $B_{k} \cdot k=0$, we can write

$$
\begin{aligned}
\operatorname{div}\left(v \otimes w_{o}\right) & =w_{o} \cdot \nabla v+\left(\operatorname{div} w_{o}\right) v \\
& =\sum_{|k|=\lambda_{0}}\left[a_{k}\left(B_{k} \cdot \nabla\right) v+v\left(B_{k} \cdot \nabla a_{k}\right)\right] e^{i \lambda k \cdot x} .
\end{aligned}
$$


The claim follows from Corollary 5.3 with $m=1$.

\section{Conclusion: proof of Proposition 2.2}

In this section we collect the estimates from the preceding sections. For simplicity we set

$$
\mu=\lambda^{\beta} .
$$

It should be noted, however, that due to the requirement (18) we can only ensure $\mu \sim \lambda^{\beta}$ for large $\lambda$.

We claim that for an appropriate choice of $\alpha$ and $\beta$, the estimates (9), (10), (11) and (12) will be satisfied for sufficiently large $\lambda$. First of all recall that, by the choice of $M$ we have

$$
\left\|w_{o}\right\|_{0} \leq \frac{\sqrt{M \delta}}{2}
$$

(cf. with (30)) and $M>1$. Therefore (11) follows from the estimate $\left\|w_{c}\right\|_{\alpha} \leq$ $C \mu \lambda^{\alpha-1}$ (cf. with (47)) if, for instance, we can prescribe

$$
C \frac{\mu}{\lambda^{1-\alpha}}=C \lambda^{\alpha+\beta-1} \leq \frac{\sqrt{\delta}}{2} .
$$

On the other hand (12) follows easily from (56) and the identity $p_{1}-p=$ $-\frac{1}{2}\left|w_{o}\right|^{2}$.

Also, from (49) it follows that (9) is satisfied provided

$$
C \lambda^{\alpha+\beta-1} \leq \frac{1}{8} \delta \min _{t \in[0,1]} e(t) .
$$

Finally, (51), (52), as well as the estimates on the error (53)-(55) imply that

$$
\left\|\stackrel{\circ}{R}_{1}\right\|_{\alpha} \leq C\left(\lambda^{\alpha-\beta}+\lambda^{\alpha+2 \beta-1}+\lambda^{2 \alpha+\beta-1}\right) .
$$

Therefore, any choice of $\alpha, \beta$ such that

$$
\alpha<\beta, \quad \alpha+2 \beta<1
$$

will ensure that (9), (10), (11) and (12) will be valid for sufficiently large $\lambda$. This completes the proof. As a side remark observe that (57) requires $\alpha<\frac{1}{3}$.

Acknowledgements We wish to thank Peter Constantin and Sergio Conti for several very valuable discussions on earlier attempts to prove Theorem 1.1. Moreover we are grateful to Antoine Choffrut for several comments on earlier versions of the paper, which considerably improved its readability. The first author acknowledges the support of the SFB Grant TR71, the second author acknowledges the support of the ERC Grant Agreement No. 277993 and the support of the Hausdorff Center for Mathematics in Bonn. 


\section{References}

1. Chiodaroli, E.: A counterexample to well-posedness of entropy solutions to the incompressible Euler system. Preprint (2012)

2. Constantin, P., Majda, A.: The Beltrami spectrum for incompressible fluid flows. Commun. Math. Phys. 115(3), 435-456 (1988)

3. Constantin, P.E.W., Titi, E.S.: Onsager's conjecture on the energy conservation for solutions of Euler's equation. Commun. Math. Phys. 165(1), 207-209 (1994)

4. Conti, S., De Lellis, C., Székelyhidi, L. Jr.: $h$-principle and rigidity for $C^{1, \alpha}$ isometric embeddings. In: Proceedings of the Abel Symposia, vol. 7, pp. 83-116 (2012). doi:10.1007/ 978-3-642-25361-4_5

5. Cordoba, D., Faraco, D., Gancedo, F.: Lack of uniqueness for weak solutions of the incompressible porous media equation. Arch. Ration. Mech. Anal. 200(3), 725-746 (2011)

6. De Lellis, C., Székelyhidi, L. Jr.: The Euler equations as a differential inclusion. Ann. Math. (2) 170(3), 1417-1436 (2009)

7. De Lellis, C., Székelyhidi, L. Jr.: On admissibility criteria for weak solutions of the Euler equations. Arch. Ration. Mech. Anal. 195(1), 225-260 (2010)

8. De Lellis, C., Székelyhidi, L. Jr.: The $h$-principle and the equations of fluid dynamics. Preprint (2011)

9. Duke, W.: An introduction to the Linnik problems. In: Equidistribution in Number Theory, an Introduction. NATO Sci. Ser. II Math. Phys. Chem., vol. 237, pp. 197-216. Springer, Dordrecht (2007)

10. Eliashberg, Y., Mishachev, N.: Introduction to the $h$-principle. Graduate Studies in Mathematics, vol. 48. American Mathematical Society, Providence (2002)

11. Eyink, G.L.: Energy dissipation without viscosity in ideal hydrodynamics. I. Fourier analysis and local energy transfer. Physica D 78(3-4), 222-240 (1994)

12. Gilbarg, D., Trudinger, N.S.: Elliptic Partial Differential Equations of Second Order. Classics in Mathematics. Springer, Berlin (2001). Reprint of the 1998 edition

13. Gromov, M.: Partial Differential Relations. Ergebnisse der Mathematik und ihrer Grenzgebiete (3), vol. 9. Springer, Berlin (1986)

14. Iwaniec, H.: Fourier coefficients of modular forms of half-integral weight. Invent. Math. 87(2), 385-401 (1987)

15. Kirchheim, B., Müller, S., Šverák, V.: Studying nonlinear PDE by geometry in matrix space. In: Hildebrandt, S., Karcher, H. (eds.) Geometric Analysis and Nonlinear Partial Differential Equations, pp. 347-395. Springer, Berlin (2003)

16. Linnik, Y.V.: Ergodic Properties of Algebraic Fields. Ergebnisse der Mathematik und ihrer Grenzgebiete, vol. 45. Springer, New York (1968). Translated from the Russian by M.S. Keane

17. Nash, J.: $C^{1}$ isometric imbeddings. Ann. Math. 60, 383-396 (1954)

18. Onsager, L.: Statistical hydrodynamics. Nuovo Cimento (9) 6(Suppl. 2), 279-287 (1949). (Convegno Internazionale di Meccanica Statistica)

19. Sarnak, P.: Some Applications of Modular Forms. Cambridge Tracts in Mathematics, vol. 99. Cambridge University Press, Cambridge (1990)

20. Scheffer, V.: An inviscid flow with compact support in space-time. J. Geom. Anal. 3(4), 343-401 (1993)

21. Shnirelman, A.: On the nonuniqueness of weak solution of the Euler equation. Commun. Pure Appl. Math. 50(12), 1261-1286 (1997)

22. Shnirelman, A.: Weak solutions with decreasing energy of incompressible Euler equations. Commun. Math. Phys. 210(3), 541-603 (2000)

23. Shvydkoy, R.: Convex integration for a class of active scalar equations. J. Am. Math. Soc. 24(4), 1159-1174 (2011)

24. Simon, L.: Schauder estimates by scaling. Calc. Var. Partial Differ. Equ. 5(5), 391-407 (1997) 
25. Sychev, M.A.: A few remarks on differential inclusions. Proc. R. Soc. Edinb., Sect. A 136(3), 649-668 (2006)

26. Székelyhidi, L. Jr.: Relaxation of the incompressible porous medium equation. Ann. Sci. l'ENS 45(3), 491-509 (2012)

27. Székelyhidi, L. Jr., Wiedemann, E.: Young measures generated by ideal incompressible fluid flows. doi:10.1007/s00205-012-0540-5

28. Wiedemann, E.: Existence of weak solutions for the incompressible Euler equations. Ann. Inst. Henri Poincaré, Anal. Non Linéaire 28(5), 727-730 (2011) 